\title{
Determinação de um Tamanho Ótimo Amostral para a Análise do Desempenho de Estudantes em Ambientes Educacionais Ubíquos
}

\author{
Title: Determination of an Optimal Sample Size for Student Performance Analysis in Ubiquitous \\ Learning Environments \\ Taffarel Brant-Ribeiro \\ Instituto Federal de Educação, Ciência e Tecnologia do Sul de Minas Gerais, \\ Campus Passos. \\ Passos, MG, Brasil. \\ brant.ribeiro@ifsuldeminas.edu.br \\ Renan Gonçalves Cattelan \\ Universidade Federal de Uberlândia, \\ Faculdade de Computação. \\ Uberlândia, MG, Brasil. \\ renan@ufu.br
}

Resumo Dispositivos computacionais se tornaram elementos onipresentes que passaram a fazer parte do cotidiano humano. Este paradigma atual de interatividade é denominado Computação Ubíqua e seu emprego no âmbito acadêmico permite automatizar atividades pedagógicas por meio da criação e implementação de Ambientes Educacionais Ubíquos. Na literatura, há diversos trabalhos que analisam estas tecnologias; no entanto eles focam unicamente em realizar pesquisas para concluir se a utilização de novas ferramentas gera melhorias em variáveis observadas, não dedicando-se também em analisar inicialmente se o tamanho das amostras utilizadas é robusto o suficiente para garantir confiabilidade nos resultados alcançados. Tais análises podem resultar em informações inexatas, devido à falta de precisão experimental dos métodos empregados nos estudos. Diversos fatores influenciam a precisão e confiabilidade experimentais, dentre eles a heterogeneidade do material experimental sob análise. Para se reduzir o erro experimental, é indispensável utilizar amostras com dimensões adequadas e capazes de compreender as características das populações observadas. Neste contexto, esta pesquisa determinou um tamanho ótimo amostral de 25 indivíduos para a análise do desempenho de estudantes que não utilizam tecnologias educacionais e de 20 alunos para turmas que possuem contato com ferramentas educacionais durante os semestres letivos. Tais resultados se mostram relevantes para pesquisas que fazem uso de fatores humanos em suas análises, pois demonstram que amostras com quantidades reduzidas de observações mostram-se capazes de compreender uma fatia relevante do comportamento das variáveis sob investigação.

Palavras-Chave: Tamanho Ótimo de Amostra; Learning Analytics; Ambientes Educacionais Ubiquos; Computação Ubíqua.

\begin{abstract}
Computational devices have become ubiquitous elements in everyday human life. This current paradigm of interactivity is called Ubiquitous Computing and its usage in academic scope allows the automation of educational activities through the creation and implementation of Ubiquitous Educational Environments. In the literature, several studies analyze these technologies, however they are focused solely on conducting researches to conclude if the usage of new tools leads to improvements in observed variables, not focusing also on initially analyzing if the sample size is sufficient to ensure reliable results. These analyzes may result in inaccurate information, due to the lack of experimental accuracy of the methods used in the studies. Several factors influence experimental precision and reliability, such as heterogeneity of the experimental material under analysis. In order to reduce experimental error, it is essential to use samples with adequate dimensions and that are able to comprehend the observed populations' characteristics. In this context, this research determined an optimal sample size of 25 students to analyze the performance of students which do not use educational technologies and of 20 students for classes that are in touch with educational systems during academic terms. These results are relevant to researches that make use of human factors, since we demonstrate that samples with reduced amounts of observations are able to comprise most part of the behavior of variables under analysis.
\end{abstract}

Keywords: Optimal Sample Size; Learning Analytics; Ubiquitous Learning Environments; Ubiquitous Computing. 


\section{Introdução}

Dispositivos computacionais têm se propagado de maneira intensa na sociedade, tornando-se cada vez mais comuns e transformando-se em elementos onipresentes que entrelaçaram-se por completo ao modo de vida das pessoas [1]. Este paradigma atual de interação entre humanos e computadores é denominado Computação Ubíqua (UbiComp), e sua utilização possibilita que os usuários sejam auxiliados por equipamentos tecnológicos de modo transparente durante o exercício de suas tarefas do dia a dia [2].

Ao se realizar a instrumentação de ambientes predeterminados - como áreas domésticas, empresariais ou educacionais - com dispositivos de UbiComp, aborda-se uma vertente de pesquisa intitulada Captura e Acesso $(C \& A)$. Esse campo versa, essencialmente, sobre a possibilidade de se capturar fluxos de conteúdos apresentados em determinados locais e disponibilizá-los para indivíduos interessados, de modo que os usuários envolvidos possam acessar as informações capturadas no momento em que preferirem.

No escopo educacional, a UbiComp permite realizar a automatização de processos pedagógicos, contribuindo de maneira contínua com atividades de ensino/aprendizagem ao equipar salas de aula com dispositivos tecnológicos e criar Ambientes Educacionais Ubíquos (AEUs) [3, 4]. $A E U s$ podem ser instrumentados com lousas eletrônicas, câmeras digitais e microfones, permitindo a produção de artefatos multimídia capazes de reproduzir posteriormente as experiências vividas em sala de aula [5]. Os alunos podem, deste modo, concentrar sua atenção na experiência da aula e na aprendizagem, certos de que os detalhes serão registrados e estarão disponíveis para posterior acesso. Deste modo, o emprego de $A E U s$ permite realizar a integração de ambientes reais e virtuais de aprendizagem, eliminando limites físicos das salas de aula e abordando uma vertente de pesquisa intitulada Aprendizagem Ubíqua (U-Learning), que preza pela possibilidade de se elaborar, distribuir e obter conhecimento em qualquer local e circunstância [6].

AEUs estão intrinsecamente ligados aos conceitos de UbiComp e $C \& A$, pois permitem que atividades acadêmicas sejam gravadas no momento em que ocorrem e permitem que alunos e professores possam acessá-las posteriormente. No entanto, ao se trabalhar com sistemas educacionais, diversas questões despontam sobre a eficiência dessas tecnologias e quais benefícios elas propiciam de fato ao escopo acadêmico. Trabalhos neste âmbito abrangem uma linha de pesquisa denominada Learning Analytics (LA), compreendida como o processo de se coletar e analisar dados de discentes para estimar seus progressos acadêmicos e antever desempenhos futuros [7, 8]. O emprego da $L A$ também fornece subsídios de caráter essencial para atividades de projeto, desenvolvimento e refinação de tecnologias educacionais, empregando métodos de engenharia capazes de levantar requisitos funcionais e não-funcionais de software e criando heurísticas específicas para a manutenção de sistemas no escopo acadêmico.

Tendo sido formalizada inicialmente em 2010, a área de $L A$ é uma temática de pesquisa contemporânea, originada a partir da interseção de diversas vertentes científicas, tais como a Psicologia, a Ciência da Computação, a Pedagogia e a Ciência da Aprendizagem [9, 10, 11]. Em razão disso, ela possui como intuito elaborar e investigar técnicas para se recuperar e analisar informações educacionais, empregando procedimentos oriundos de diversos campos científicos, tais como a Estatística e a Visualização de Dados.

Conforme Chatti et al. [8], o processo de aprendizagem tem se expandido em largas escalas, abordando, inclusive, variáveis de tempo, espaço e mídias utilizadas. Consequentemente, grandes volumes de dados a respeito de indivíduos em atividades de assimilação de conhecimento têm sido gerados de maneira contínua. Tais dados podem ser interpretados como rastros que as pessoas deixam quando interagem com ambientes educacionais e utilizados para se compreender como esse fenômeno tende a acontecer e como otimizar processos de aprendizagem.

Por se tratar de uma temática de pesquisa contemporânea, a área de $L A$ ainda não dispõe completamente de um arcabouço bem fundamentado de métodos, heurísticas e padrões para a realização e replicação de pesquisas científicas. Na literatura, trabalhos sobre desempenho e adaptabilidade de usuários são frequentemente observados e abrem margem para diversas análises, como a verificação de ganhos para estudantes que fazem uso de ferramentas acadêmicas $[4,12,13]$. No entanto, a maioria dessas pesquisas preocupa-se apenas em fazer comparações entre usuários, expondo que existem aumentos de desempenho entre grupos observados, mas não dedicando-se em averiguar inicialmente se o tamanho das amostras analisadas mostra-se robusto o suficiente para representar com alto nível de confiabilidade as populações das quais são retiradas.

Diversos fatores influenciam a precisão e a confiabilidade experimentais, dentre eles o nível de heterogeneidade dos ambientes e do material experimental sob análise $[14,15]$. Para se diminuir o erro experimental, é imprescindível utilizar amostras com dimensões adequadas e capazes de suprir as características das populações observadas. Dentre os métodos existentes na literatura para 
estimar o tamanho ótimo de amostras, o Método de Máxima Curvatura Modificado (MMCM), apresentado por Lessman \& Atkins [16] e Meier \& Lessman [17], é bastante empregado por fornecer resultados precisos ao estabelecer uma equação de regressão para explicar a relação entre os coeficientes de variação e seus devidos tamanhos de amostra [18]. Tal método também destaca-se por ser capaz de ilustrar num plano cartesiano os coeficientes de variação das amostras junto a seus respectivos tamanhos, realizando o cálculo do valor da abcissa referente ao ponto exato onde ocorre a máxima curvatura do modelo, indicando o tamanho ótimo da amostra analisada.

Com essas assertivas em mente, esta pesquisa objetivou determinar e explorar o tamanho ótimo de amostra para a análise do desempenho de estudantes em contato com $A E U$ s. O presente trabalho estendeu a contribuição apresentada inicialmente em Brant-Ribeiro \& Cattelan [19], realizando contextualizações mais sólidas acerca do problema de pesquisa estudado, descrição do $A E U$ empregado no decorrer da investigação, detalhamento do fluxo metodológico e aumento das turmas analisadas durante a pesquisa. Além disso, a estrutura e o conteúdo do trabalho foram refinados por meio do acréscimo de novas figuras e aprimoramento de resultados, discussões e considerações finais.

No decorrer desta investigação, enxergou-se também a necessidade de se determinar o tamanho ótimo para investigar alunos de graduação que não estão em contato com tecnologias educacionais, de modo a ser possível traçar um paralelo entre os resultados obtidos e se recomendar uma estimativa capaz de compreender ambas as situações. Tais informações se mostram essenciais para basearem pesquisas na vertente de $L A$, de modo a delimitar um tamanho mínimo de amostra capaz de representar as populações investigadas com alto nível de confiabilidade. Para esta finalidade, o restante deste artigo está estruturado da seguinte maneira: na Seção 2, o $A E U$ utilizado nesta pesquisa é detalhado; na Seção 3, material e métodos são demonstrados; na Seção 4, resultados são apresentados e discutidos; na Seção 5, trabalhos relacionados são revisados e, por fïm, considerações finais são pontuadas na Seção 6 .

\section{Classroom eXperience}

O Classroom eXperience $(C X)^{1}$ é um $A E U$ desenvolvido com o intuito de registrar os fluxos de conteúdo multimídia apresentados em sala de aula e disponibilizálos posteriormente para professores e estudantes [20, 21]. Por meio de sua utilização, é possível capturar, armazenar, acessar e estender as mídias geradas em ambientes

\footnotetext{
${ }^{1} \mathrm{http}: / / \mathrm{cx}$. facom.ufu.br/
}

educacionais instrumentados e contribuir com as atividades de ensino/aprendizagem de usuários que utilizam esta tecnologia no cotidiano $[22,23]$.

O processo de captura do $C X$ se baseia nas quatro fases essenciais da $C \& A$ : "pré-produção" (momento no qual os conteúdos são preparados), "gravação ao vivo" (quando as atividades acadêmicas são gravadas no momento em que acontecem), "pós-produção" (fase em que os fluxos produzidos são armazenados) e "acesso" (momento em que os conteúdos capturados são disponibilizados para alunos e professores) [24].

Além destas fases, uma quinta etapa intitulada “extensão" também pode ser destacada das supracitadas [25]. Tal etapa compreende o momento em que os usuários complementam e enriquecem os conteúdos capturados por meio de atividades interativas sociais e colaborativas com a plataforma educacional. Para que esses processos ocorram de maneira transparente, o $C X$ atualmente conta com duas salas de aula equipadas com dispositivos computacionais (como microfones, lousas digitais, câmeras e projetores multimídia), localizadas na Faculdade de Computação da Universidade Federal de Uberlândia (FACOM/UFU), conforme pode ser visto na Figura 1.

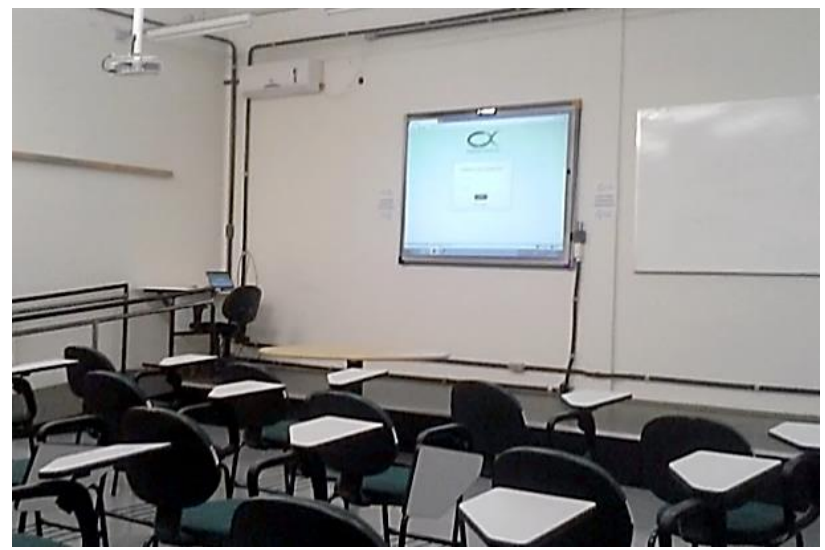

Figura 1: Sala de aula instrumentada com dispositivos de UbiComp (Fonte: Adaptado de Brant-Ribeiro; Biase \& Cattelan [22]).

Demandando modificações mínimas na maneira com que as aulas convencionais são lecionadas, o $C X$ emprega recursos da UbiComp para auxiliar tanto professores, enquanto realizam a captura das aulas, quanto alunos, durante atividades de acesso ao conteúdo capturado. Para isso, o sistema conta com componentes de hardware e software especializados para realizar a gravação das atividades educacionais, sincronizar automaticamente os fluxos de mídia concebidos e disponibilizar esse conteúdo por meio de uma interface de acesso $W e b$, cujas telas podem ser observadas na Figura 2. 
(a)

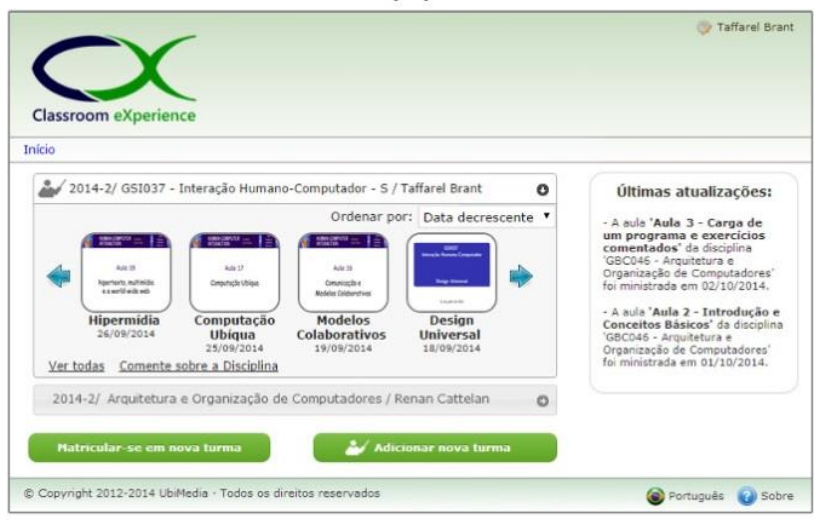

(b)

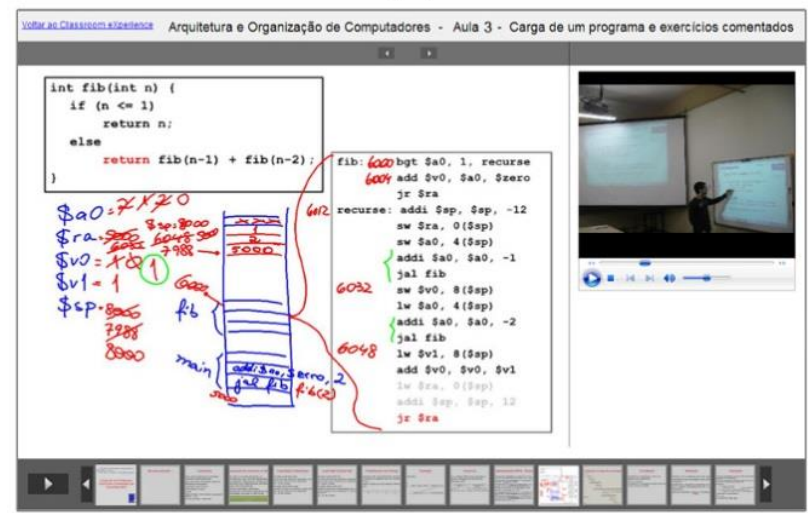

Figura 2: Interface Web do CX: (a) home page da plataforma e (b) tela de aula capturada (Fonte: Brant-Ribeiro \& Cattelan [19]).

Ao logarem no sistema, os usuários são direcionados para a home page da plataforma (Figura 2(a)), na qual é possível, para um professor, cadastrar uma nova turma e, para os alunos, matricularem-se nas turmas já cadastradas no $C X$. Além disso, esta página também exibe as últimas atualizações que aconteceram no sistema, tais como aulas capturadas recentemente e avaliações registradas pelos professores - como provas, trabalhos, exercícios e seminários. De modo a melhorar a organização desses dados, eles são exibidos empregando-se filtros temporais e de disciplinas vinculadas aos usuários do $C X$, de maneira que apenas alunos matriculados em turmas nas quais essas atualizações acontecem são capazes de enxergar tais informações na home page.

Ao selecionarem uma aula específica, os usuários devem escolher em qual formato de apresentação o conteúdo capturado deverá ser exibido. No $C X$, atualmente há três tipos de formato de apresentação possíveis: somente slides, somente vídeo e completo. Caso selecione a primeira opção, o usuário será direcionado para uma página contendo os slides utilizados pelo professor durante uma sessão de captura, já com as anotações realizadas nessas mídias. A segunda opção apresentará apenas o vídeo de uma aula capturada pelo sistema e a terceira exibirá ambos os fluxos de mídia, conforme a Figura 2(b).

\section{Material e Métodos}

Para realizar esta pesquisa, foram utilizados dados provenientes de dez turmas que cursaram disciplinas da grade curricular dos cursos de graduação da FA$\mathrm{COM} / \mathrm{UFU}$. Dessas, cinco não tiveram qualquer contato com a plataforma educacional $C X$, ao passo que as demais utilizaram o $A E U$ normalmente no decorrer do semestre letivo. Objetivando compreender estudantes tanto do curso de graduação em bacharelado em Sistemas de Informação quanto em Ciência da Computação, foram selecionadas disciplinas que ambos os cursos possuem em suas respectivas grades curriculares, de modo a propositalmente haver amostras mistas com quantidades diversificadas de estudantes de cada curso.

As dez amostras selecionadas foram compostas por $\mathrm{N}$ observações da variável sob análise. Tendo em vista que cada amostra compreendeu uma turma de alunos matriculados numa respectiva disciplina, o tamanho das amostras não se manteve fixo e apresentou variações entre 26 e 44 estudantes. Como a variável estudada nesta pesquisa foi o desempenho acadêmico dos alunos, as notas dos estudantes foram coletadas ao final de cada semestre letivo e, para cada uma das amostras resultantes, foram realizadas combinações aleatorizadas (reamostragens) das observações para formar subamostras de diversos tamanhos a partir da amostra original.

Para a realização do processo de reamostragem, o método Bootstrapping [26] foi empregado nesta fase. Deste modo, para cada subamostra de tamanho $n$ originada de uma amostra de tamanho $N$, foram realizadas 2000 reamostragens para todo $n \leq N$. Em seguida, para cada um dos conjuntos de 2000 subamostras de mesmo tamanho resultantes, realizou-se o cálculo da média $(\bar{x})$, variância $\left(s^{2}\right)$, desvio padrão $(s)$ e coeficiente de variação $(C V)$, além de um intervalo de confiança (IC) de $95 \%$ para essas medidas.

Para a realização do cálculo do tamanho ótimo amostral por meio do Método de Máxima Curvatura Modificado $(M M C M)$, representou-se inicialmente a relação entre cada $C V$ e seu conjunto de 2000 subamostras de mesmo tamanho empregando-se a equação de regressão de potência (1):

$$
C V_{x}=a X^{-b}
$$


na qual: $C V_{x}$ é o coeficiente de variação entre as subamostras de tamanho $n ; a$ é o coeficiente de variação de amostras de tamanho unitário; $X$ é o tamanho das subamostras; e $b$ é o coeficiente de regressão [27].

De modo a elucidar os parâmetros $a$ e $b$ utilizados na equação de regressão (1): o coeficiente de variação unitário $a$ indica o ponto no qual a linha de regressão intercepta o eixo $y$ utilizando valores de $x$ e $y$ existentes, referentes aos dados coletados para a execução da pesquisa. Já o coeficiente de regressão $b$ retrata a taxa de mudança ao longo da linha de regressão, ou seja, representa o nível de variabilidade das amostras sob análise.

Também realizou-se o cálculo do coeficiente de determinação $\left(R^{2}\right)$ para cada turma analisada no decorrer da pesquisa, utilizando-se a fórmula (2):

$$
R^{2}=\frac{\sum_{i=1}^{n}\left(\hat{y}_{i}-\bar{y}\right)^{2}}{\sum_{i=1}^{n}\left(y_{i}-\bar{y}\right)^{2}}
$$

na qual: $\hat{y}_{i}$ é o valor estimado de cada $y_{i}$ e $\bar{y}$ é a média das observações $y$ de cada amostra.

A fórmula de cálculo do $R^{2}$ emprega, em seu numerador, a diferença entre a média das observações da amostra e o valor estimado de cada observação, realizando o somatório de seus quadrados. Em seu denominador, é calculada a soma dos quadrados das diferenças entre a média das observações e cada valor observado na amostra, resultando na soma total dos quadrados. O coeficiente de determinação indica, portanto, quanto o modelo empregado mostra-se capaz de explicar as observações obtidas nas amostras, permitindo compreender o quão explicativo é determinado modelo e o quanto ele se ajusta em relação aos valores observados [28].

Como a fórmula (2) resulta em um valor que varia entre zero e um, este resultado foi convertido para porcentagem. Além disso, embora quanto maior o $R^{2}$, melhor seja o ajuste da função aos dados, um $R^{2}$ acima de $70 \%$ já indica um bom ajuste para os modelos utilizados.

Por fim, representou-se a relação entre os $C V S$ e os tamanhos das subamostras de maneira gráfica em eixos cartesianos, nos quais o eixo $y$ representou o $C V$ e o eixo $x$ retratou o intervalo dos tamanhos de amostra. Após estabelecer as estimativas dos $C V S$ de todas as subamostras de cada turma analisada, calculou-se o valor referente ao ponto da abcissa no qual ocorre a máxima curvatura do modelo utilizado, correspondente ao tamanho ótimo de amostra, denominado $X_{M C}$ e obtido por meio da fórmula (3):

$$
X_{M C}=\left[a^{2} b^{2}(2 b+1) / b+2\right]^{(1 / 2 b+2)}
$$

na qual: $X_{M C}$ é o valor exato da abcissa que corresponde ao ponto de máxima curvatura do modelo; $a$ é o coeficiente de variação de amostras de tamanho unitário; e $b$ é o coeficiente de regressão [17].

Em razão da fórmula supracitada resultar num valor com casas decimais e, para a presente pesquisa, o tamanho ótimo precisar ser um valor inteiro, realizou-se o arredondamento de cada $X_{M C}$ obtido, respeitando a regra adotada pela ABNT [29]. Por fim, conforme Meier \& Lessman [17], os tamanhos ótimos resultantes a partir do $M M C M$ devem ser expressos numa medida denominada Unidade Básica $(U B)$, que demanda transformações quando uma $U B$ compreende um conjunto de observações maior que um. Neste trabalho, cada $U B$ representou exatamente um estudante, de modo a não ser necessário realizar outras transformações nos tamanhos obtidos além de arredondamentos.

\section{Resultados e Discussões}

Durante a realização do Bootstrapping, as subamostras com tamanhos muito reduzidos $(n<5)$ apresentaram dispersões de caráter desuniforme e inconclusivo. Tal comportamento já era esperado, pois necessita-se que haja um número mínimo de observações para ser possível analisar a distribuição das amostras. Deste modo, estabeleceu-se um limite mínimo de cinco observações em cada amostra para o início da análise dos $C V s$ nas turmas examinadas. Essa análise se estendeu até o tamanho original das dez amostras selecionadas para este estudo.

A Figura 3 apresenta os dez eixos cartesianos correspondentes às amostras analisadas, sendo os cinco dispostos na primeira coluna (gráficos de $\mathrm{A}$ a $\mathrm{E}$ ) referentes às turmas que não tiveram contato com o $C X$ e os demais eixos (gráficos de $\mathrm{F}$ a $\mathrm{J}$ ) relativos às turmas que empregaram o sistema durante o semestre letivo. Os $C V s$ exatos de cada tamanho de subamostra foram representados em seus respectivos eixos junto à equação de regressão de potência que indicou a tendência dos dados obtidos. Também calcularam-se os limites superiores e inferiores dos $C V s$, optando-se por representá-los já aplicados ao modelo, de modo a demonstrar os pontos máximos e mínimos que os $C V s$ seriam capazes de alcançar em cada tamanho de subamostra sob um $I C$ de $95 \%$. 

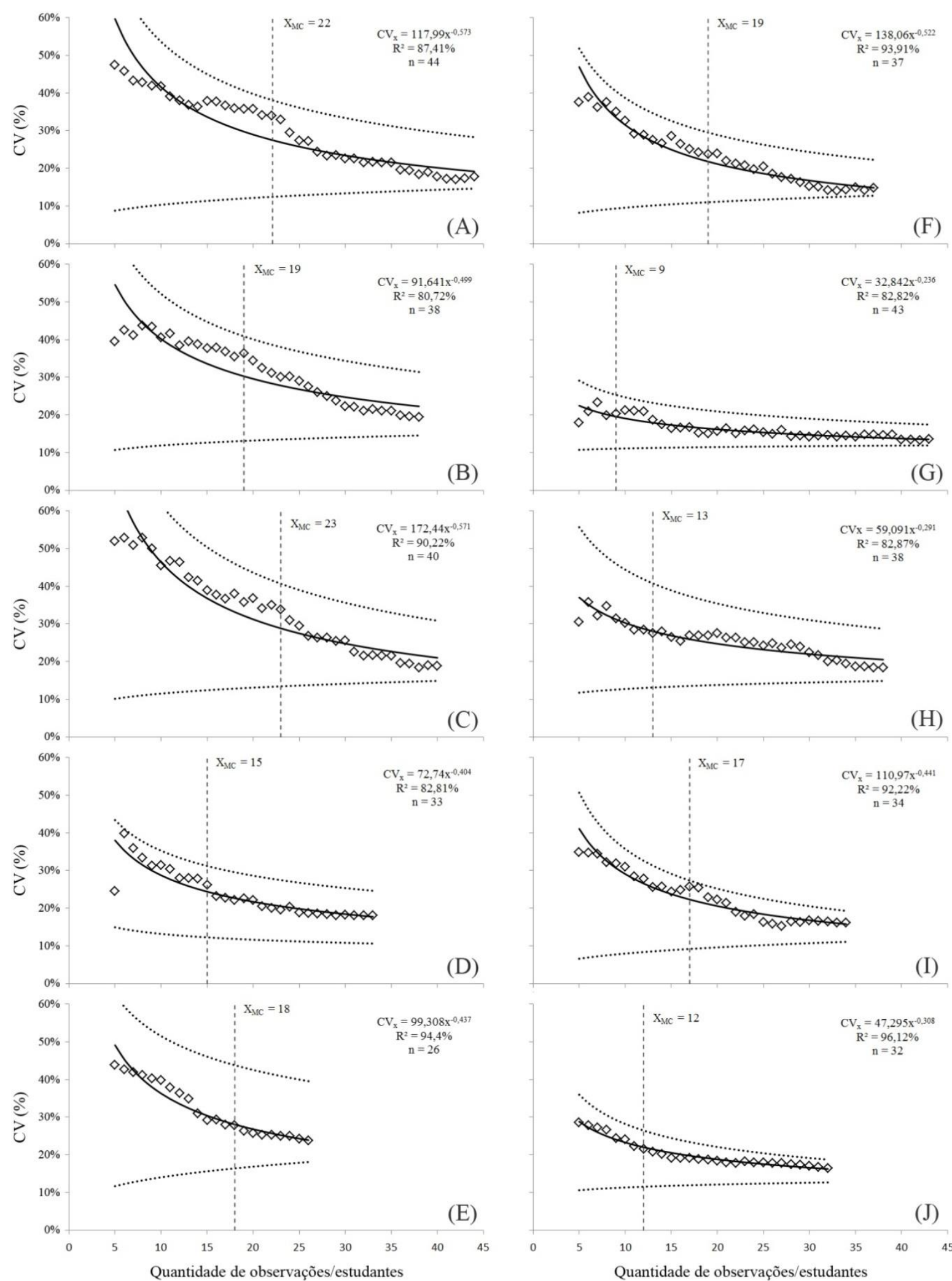

$\diamond$ Coeficiente de Variação (CV) - Regressão $C V_{x}=a X^{-b}$

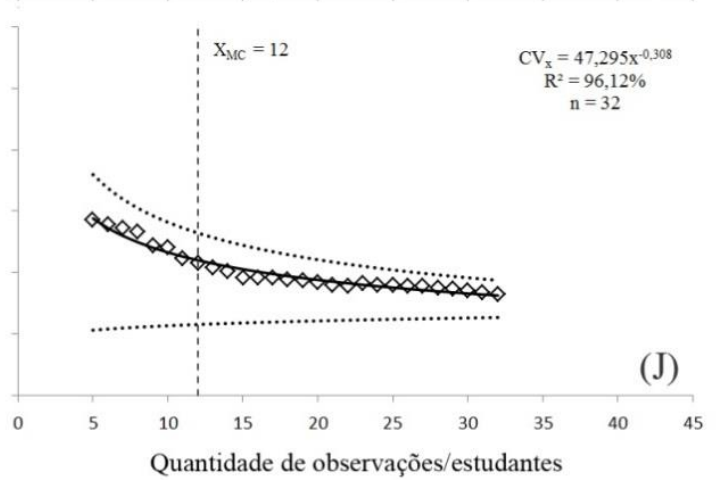

Figura 3: Relações entre os coeficientes de variação e os tamanhos de subamostras para a variável desempenho dos estudantes em dez turmas de cursos de computação. 
A partir da análise das dez turmas utilizadas, foi possível perceber que o valor do $C V$ diminuiu ao passo que o tamanho das subamostras aumentou. Por meio do $M M C M$, o tamanho ótimo de amostra obtido entre as cinco turmas que não tiveram contato com o $C X$ variou entre 15 e 22 estudantes, ao passo que nas turmas que empregaram o sistema durante os semestres letivos a estimativa foi de 9 a 19 alunos.

Com base nesses resultados, torna-se possível compreender que as turmas que utilizaram o $A E U$ no decorrer dos semestres letivos tenderam a apresentar pontos de máxima curvatura inferiores aos obtidos naquelas que não tiveram contato com a tecnologia. Isso demonstra que quantidades de observações menores que as originalmente existentes nas amostras já teriam sido suficientes para representar o comportamento da variável sob análise. Além disso, como entre as turmas que utilizaram o $C X$ o nível médio de variabilidade das notas foi ainda menor que naquelas que não utilizaram o sistema, é possível ratificar a hipótese de que maiores índices de nivelamento entre o desempenho dos alunos podem ocorrer em razão dos mesmos passarem a estudar os conteúdos das disciplinas a partir de uma fonte em comum, conforme presumido em Brant-Ribeiro; Biase \& Cattelan [22].

Como o MMCM possibilita o cálculo do valor da abcissa referente ao ponto onde ocorre a máxima curvatura do modelo, e isso indica o tamanho ótimo de amostra para se analisar uma determinada variável, também é necessário propor um tamanho capaz de abranger todas as amostras que contenham observações dessa variável. Em função disso, como as turmas que utilizaram o $C X$ apresentaram tamanhos que variaram entre 9 e 19 alunos, infere-se que o tamanho ótimo de amostra capaz de analisar o desempenho de alunos em contato com tecnologias educacionais seja de pelo menos 20 indivíduos. Do mesmo modo, como os tamanhos obtidos entre as turmas que não tiveram contato com o $A E U$ variaram entre 15 e 22 alunos, propõe-se que para se investigar turmas que não utilizam sistemas educacionais, no mínimo sejam observados 25 estudantes.

Além disso, por meio da análise das linhas de tendência presentes nos eixos da Figura 3, também foi possível perceber que a diminuição do $C V$ não apresentou um comportamento proporcional ao aumento dos tamanhos das subamostras, acentuando-se no início e tornando-se mais próxima à estabilidade em quantidades mais elevadas de observações. Deste modo, caso as amostras analisadas tivessem sido ainda maiores, pressupõe-se que se alcançaria um ponto em que as linhas de tendência dos limites superior e inferior e do $C V$ acabariam se interceptando. Por meio desse comportamento, é possível deduzir que o aumento do tamanho das amostras apenas se manteve conveniente até determinado ponto, a partir do qual o emprego de quantidades ainda maiores de observações não foi compensatório, em função dos baixos ganhos em precisão experimental.

Por fim, o $R^{2}$ alcançado nas análises manteve-se acima de $80 \%$ em todas as amostras estudadas, o que demonstra boa precisão de ajuste das regressões e alta confiabilidade nas estimativas obtidas nesta pesquisa. $\mathrm{O}$ coeficiente de regressão $b$, capaz de representar a variabilidade dos experimentos sob análise, variou de 0,404 a 0,573 entre as cinco turmas que não tiveram contato com o $C X$ e de 0,236 a 0,522 nas turmas que o empregaram durante seus respectivos semestres letivos (Tabela 1).

Tabela 1: Coeficientes de variação unitários e de regressão obtidos a partir da analise das dez amostras utilizadas na pesquisa.

\begin{tabular}{ccc}
\hline Amostra $^{1}$ & Parâmetro $\boldsymbol{a}$ & Parâmetro $\boldsymbol{b}$ \\
\hline $\mathbf{A}$ & 117,99 & $0,573^{*}$ \\
\hline $\mathbf{B}$ & 91,641 & 0,499 \\
\hline $\mathbf{C}$ & 172,44 & 0,571 \\
\hline $\mathbf{D}$ & 72,74 & 0,404 \\
\hline $\mathbf{E}$ & 99,308 & 0,437 \\
\hline $\mathbf{F}$ & 138,06 & 0,522 \\
\hline $\mathbf{G}$ & 32,842 & $0,236^{* *}$ \\
\hline $\mathbf{H}$ & 59,091 & 0,291 \\
\hline $\mathbf{I}$ & 110,97 & 0,441 \\
\hline $\mathbf{J}$ & 47,295 & 0,308 \\
\hline
\end{tabular}

${ }^{1}$ Amostras de $\mathrm{A}$ a $\mathrm{E}$ representam turmas que não tiveram contato com o $\mathrm{CX}$; Amostras de $\mathrm{F}$ a J representam turmas que empregaram o CX no decorrer dos semestres letivos; Parâmetro $a$ : Coeficiente de variação de amostras de tamanho unitário; Parâmetro $b$ : Coeficiente de regressão das amostras; *: Maior valor de $b$ obtido entre as amostras; **: Menor valor de $b$ obtido entre as amostras.

Conforme apresentado na Tabela 1, os maiores valores de $b$ observados entre as turmas foram obtidos nas amostras A $(0,573)$ e C $(0,571)$, que fizeram parte do grupo de turmas que não tiveram contato com o $C X$ e apresentaram os maiores $X_{M C}$ obtidos (22 e 23, respectivamente) entre todas as turmas analisadas. Além disso, nenhuma dessas amostras apresentou um valor do parâmetro $b$ menor que 0,4 .

Tendo por base que o coeficiente de regressão $b$ representa a taxa de mudança que ocorre ao longo da curva de regressão e explicita o grau de variabilidade das amostras, tais resultados reforçam a hipótese de que as turmas que não empregaram o $C X$ no decorrer dos semestres letivos apresentaram um comportamento mais heterogê- 
neo do que aquele observado nas amostras das turmas que utilizaram o $A E U$. De fato, os menores valores de $b$ observados foram obtidos justamente nas amostras $G$ $(0,236), \mathrm{H}(0,291)$ e $\mathrm{J}(0,308)$, compreendidas entre as turmas que tiveram contato com o sistema e que obtiveram os menores $X_{M C}(9,13$ e 12 , respectivamente) dentre todas as turmas investigadas nesta pesquisa.

\section{Trabalhos Relacionados}

A determinação de um tamanho ótimo amostral compreende uma temática de pesquisa relevante, pois permite o entendimento da real dimensão da variabilidade de experimentos e a estimação de um tamanho mínimo necessário para se garantir a confiabilidade dos resultados obtidos em investigações que trabalham com amostras heterogêneas [17]. Na literatura, é possível encontrar pesquisas que determinam essa estimativa em circunstâncias específicas, tais como para melhorar o processo de classificação em atividades de mineração de dados, ajustando-se curvas de aprendizagem para determinar o tamanho de conjuntos de treinamento [30], ou estimar assintoticamente um tamanho ótimo que permita a detecção de mudanças na estatística de processos [31].

Há também trabalhos que abordam a determinação de tamanhos ótimos para o treinamento de redes neurais artificiais [32], alocação de recursos para múltiplas atividades concorrentes [33] e minimização do custo total de fabricação de circuitos digitais Ultra Large Scale Integration (ULSI) [34]. Em Confalonieri et al. [35], é apresentado o sistema SISSI, que realiza o cálculo do tamanho ótimo amostral por meio da implementação de uma técnica de reamostragem similar ao $M M C M$, denominada Jackknife. Ainda na literatura, o cálculo do tamanho ótimo amostral também é realizado para a escolha do número de experimentos a serem realizados para a compreensão de padrões diferenciais de expressão gênica [36] e para o projeto de experimentos que precisam trabalhar com amostras que sigam a distribuição exponencial, caracterizada como uma distribuição amplamente utilizada para a análise de risco e confiabilidade em bases de dados [37].

$\mathrm{Na}$ vertente de pesquisa em sistemas $W e b$, um tamanho clássico de amostra de cinco usuários foi inicialmente proposto por Nielsen [38], que justificou tal quantidade como suficiente para se compreender cerca de $80 \%$ dos problemas de usabilidade em interfaces. No entanto, esse tamanho foi readequado com a criação da Regra de $10 \pm$ 2 usuários [39] e, em seguida, ambos foram contestados por Schmettow [40], que demonstrou que a estimativa de tamanhos de amostra não era algo trivial, havendo fatores que causariam a variabilidade dos dados sob análise e sendo importante se atentar a esses detalhes para que os resultados não subestimassem as características investigadas, o que vai de encontro à necessidade de se realizar pesquisas focadas na determinação de tamanhos amostrais, tal como a apresentada neste trabalho.

No escopo educacional, tamanhos de amostra exclusivos para diferentes tipos de análise foram apresentados por Mertens [41]. Dentre as indicações, recomendou-se a utilização de pelo menos 64 participantes em testes de correlação, 15 observações em testes de regressão múltipla e um mínimo de 51 elementos para pesquisas focadas na realização de comparações, tais como analisar o impacto que novas tecnologias causam em cursos de níveis educacionais diversificados. Tais tamanhos foram obtidos por meio de análises do poder de testes estatísticos e os resultados são, na verdade, médias observadas a partir dos valores resultantes nos testes realizados para cada uma dessas vertentes [42].

Ainda nesse escopo, Slavin \& Smith [43] observaram que o tamanho das amostras tende a apresentar uma correlação inversamente proporcional ao efeito estudado em revisões sistemáticas na área educacional. Deste modo, concluíram que revisões que abordam quantidades menores de trabalhos sobre determinada temática acabam se mostrando enviesadas e relatando efeitos demasiadamente positivos do que os trabalhos que compreendem quantidades mais abrangentes de conteúdo e de fato representam o universo pesquisado.

Tais trabalhos fortalecem a relevância de se estimar tamanhos ótimos amostrais para ser possível compreender as populações investigadas em níveis satisfatórios. Pesquisas nessa vertente, no entanto, não são muito frequentes no âmbito da Ciência da Computação, devido a possibilidade de algumas áreas de atuação simularem seus estudos em quantidades elevadas para atestarem suas hipóteses. No entanto, pesquisas que envolvem elementos externos, tais como análises que observam características em fatores humanos, apresentam a necessidade de se determinar um tamanho ótimo amostral em função da impossibilidade de se trabalhar frequentemente com quantidades muito elevadas de indivíduos.

Em função disso, o presente trabalho se destaca dos observados na literatura ao demonstrar uma estimativa robusta para se analisar o desempenho acadêmico de estudantes em contato com tecnologias educacionais, tais como os AEUs. Tendo em vista que a quantidade de discentes em turmas presenciais de cursos de graduação é, muitas vezes, reduzida, mostra-se necessário estimar e trabalhar com tamanhos amostrais capazes de compreender a variabilidade das populações de alunos sob análise e permitir que resultados confiáveis sejam alcançados por pesquisadores que realizam investigações nesta vertente 
científica.

\section{Conclusões e Trabalhos Futuros}

Esta pesquisa teve como objetivo estimar o tamanho ótimo amostral para a análise do desempenho acadêmico de estudantes em cursos de graduação em bacharelado em Sistemas de Informação e Ciência da Computação. Determinou-se um tamanho ótimo de 25 alunos para a análise desta variável em turmas que não utilizam tecnologias educacionais no cotidiano e de 20 estudantes para turmas que possuem contato com tecnologias educacionais (tais como $A E U s$ ) durante os semestres letivos.

Por meio da interpretação das linhas de tendência geradas, percebeu-se que a diminuição dos $C V s$ não apresentou um comportamento proporcional à elevação dos tamanhos das amostras, intensificando-se apenas no começo e tornando-se estável à medida que as amostras tiveram suas quantidades de observações acrescidas. Em função disso, é possível compreender que a elevação do tamanho das amostras se manteve conveniente apenas até determinado ponto, a partir do qual o emprego de quantidades maiores de observações não foi compensado devido ao ganho reduzido em precisão experimental.

Este resultado reforça a pressuposição de que quantidades de observações menores que as inicialmente existentes nas amostras já teriam sido suficientes para caracterizar o comportamento da variável estudada. Deste modo, pesquisas que objetivam realizar estudos acerca do desempenho de estudantes em circunstâncias nas quais novas tecnologias são introduzidas podem empregar as estimativas obtidas neste trabalho para realizarem suas investigações com confiabilidade em suas análises.

Para uma análise ainda mais robusta acerca do desempenho acadêmico dos estudantes, recomenda-se empregar amostras de pelo menos 25 observações em ambas as vertentes demonstradas. Dessa maneira, mostra-se possível compreender a variabilidade de quaisquer turmas neste escopo e se realizar uma investigação capaz de abranger corretamente a população de interesse.

Como trabalhos futuros, pretende-se aumentar o escopo de investigação desta pesquisa, de modo a compreender dados referentes a todos os semestres letivos de cursos de graduação em Sistemas de Informação e Ciência da Computação. Dessa maneira, torna-se possível discernir quais disciplinas e momentos dos cursos tendem a apresentar maiores níveis de heterogeneidade no desempenho dos estudantes. Tais informações se mostram válidas para a identificação prévia de circunstâncias que apresentam maiores riscos de reprovação dos alunos e, consequentemente, podem levar à desistência em disciplinas matriculadas e, até mesmo, à evasão dos cursos. Desse modo, a compreensão de circunstâncias neste es- copo permitirá a criação e estudo de técnicas e propostas para a prevenção dessas situações, tais como a criação e o refinamento de ferramentas educacionais com o intuito de melhorar os processos de ensino/aprendizagem.

Estudos acerca de tamanhos amostrais se mostram relevantes para pesquisas que fazem uso de fatores humanos, pois demonstram que amostras com quantidades reduzidas de observações são capazes de compreender grande parte do comportamento da variável sob análise. Devido aos custos e obstáculos existentes para se trabalhar com um elevado número de indivíduos em contextos diversificados, o cálculo de um tamanho ótimo amostral se revela como uma alternativa robusta e válida para se obter alto índice de confiabilidade em inferências obtidas a partir de análises em contextos mais reduzidos.

\section{Agradecimentos}

Os autores gostariam de agradecer às agências de pesquisa FAPEMIG, CAPES e CNPQ, ao PET/SESu/MEC e ao Instituto Federal de Educação, Ciência e Tecnologia do Sul de Minas Gerais (IFSULDEMINAS) o apoio concedido a este trabalho.

\section{Referências}

[1] Internet World Stats. World Internet User Statistics and World Population Stats, 2016. http://www.internetworldstats.com/stats.htm. Acesso em Fevereiro/2017.

[2] M. Weiser. The Computer for the $21^{\text {st }}$ Century. Scientific American, 265(3): 94-104, 1991.

[3] X. Zhao, X. Wan e T. Okamoto. Adaptive Content Delivery in Ubiquitous Learning Environment. Em Proc. of the $6^{\text {th }}$ IEEE International Conference on Wireless, Mobile, and Ubiquitous Technologies in Education. Págs. $19-26,2010$.

[4] A. Settle, L. Dettori e M. J. Davidson. Does Lecture Capture Make a Difference for Students in Traditional Classrooms. Em Proc. of the $16^{\text {th }}$ Annual Joint Conference on Innovation and Technology in Computer Science Education. Págs. 78-82, 2011.

[5] Z. Aihua. Study of Ubiquitous Learning Environment Based on Ubiquitous Computing. Em Proceedings of the $3^{\text {rd }}$ IEEE International 
Conference on Ubi-Media Computing. Págs. 136-138, 2010.

[6] Kinshuk e S. Graf. Ubiquitous Learning. Em Encyclopedia of the Sciences of Learning. Págs. 3361-3363, 2012.

[7] A. van Barneveld, K. E. Arnold e J. P. Campbell. Analytics in Higher Education: Establishing a Common Language. EDUCAUSE Learning Initiative. (1): 1-11, 2012.

[8] M. A. Chatti, V. Lukarov, H. Thüs, A. Muslim, A. M. F. Yousef, U. Wahid, C. Greven, A. Chakrabarti e U. Schroeder. Learning Analytics: Challenges and Future Research Directions. ELearning \& Education. 10(1), 2014.

[9] S. Dawson, D. Gašević, G. Siemens e S. Joksimovic. Current State and Future Trends: a Citation Network Analysis of the Learning Analytics Field. Em Proceedins of the $4^{\text {th }}$ International Conference on Learning Analytics And Knowledge. Págs. 231-240, 2014.

[10] M. A. Chatti, A. L. Dyckhoff, U. Schroeder e H. Thüs. A Reference Model for Learning Analytics. International Journal of Technology Enhanced Learning. 4(5/6):318-331, 2012.

[11] R. Ferguson. Learning Analytics: Drivers, Developments and Challenges. International Journal of Technology Enhanced Learning. 4(5/6):304-317, 2012.

[12] M. B. Wieling e W. H. a. Hofman. The Impact of Online Video Lecture Recordings and Automated Feedback on Student Performance. Computers \& Education. 54(4):992-998, 2010.

[13] P. E. Dickson, D. I. Warshow, A. C. Goebel, C. C. Roache e W. R. Adrion. Student Reactions to Classroom Lecture Capture. Em Proceedings of the $17^{\text {th }}$ ACM Annual Conference on Innovation and Technology in Computer Science Education. Págs. 144-149, 2012.

[14] K. A. Gomez e A. A. Gomez. Statistical Procedures for Agricultural Research. $2^{\text {nd }}$ Edition. John Wiley \& Sons, 1984.

[15] R. G. D. Steel. Principles and Procedures of Statistics: A Biometrical Approach. McGrawHill, 1997.
[16] K. J. Lessman e R. E. Atkins. Optimum Plot Size and Relative Efficiency of Lattice Designs for Grain Sorghum Yield Tests. Crop Science. 3(6):477-481, 1963.

[17] V. D. Meier e K. J. Lessman. Estimation of Optimum Field Plot Shape and Size for Testing Yield in Crambe abyssinica Hochst. Crop Science. 11(5):648-650, 1971.

[18] R. L. da Silva, A. Xavier, H. G. Leite e I. E. Pires. Determinação do Tamanho Ótimo da Parcela Experimental pelos Métodos da Máxima Curvatura Modificado, do Coeficiente de Correlação Intraclasse e da Análise Visual em Testes Clonais de Eucalipto. Revista Árvore. 27(5):669-676, 2003.

[19] T. Brant-Ribeiro e R. Cattelan. Tamanho Ótimo de Amostra para Análise do Desempenho de Estudantes em Ambientes Educacionais Ubíquos. Em Anais do Simpósio Brasileiro de Informática na Educação. Págs. 31-40, 2015.

[20] H. N. M. Ferreira, R. D. Araujo, S. A. de Amo e R. G. Cattelan. Classroom Experience: A Platform for Multimedia Capture and Access in Instrumented Educational Environments. Em 2012 Brazilian Symposium on Collaborative Systems. Págs. 59-64, 2012.

[21] R. D. Araújo, T. Brant-Ribeiro, R. G. Cattelan, S. a. de Amo e H. N. M. Ferreira. Personalization of Interactive Digital Media in Ubiquitous Educational Environments. Em 2013 IEEE International Conference on Systems, Man, and Cybernetics. Págs. 3955-3960, 2013.

[22] T. Brant-Ribeiro, N. G. Biase e R. G. Cattelan. Impacto de Aplicações de Captura e Acesso em Estudantes de Cursos de Computação. Revista Brasileira de Informática na Educação. 23(2):111-126, 2015.

[23] T. Brant-Ribeiro, I. E. S. Mendonça, R. D. Araújo, M. M. Mendes, F. A. Dorça e R. G. Cattelan. Um Modelo Social e Colaborativo para Extensão de Conteúdo em Ambientes Educacionais Ubíquos. Tecnologias, Sociedade e Conhecimento. 2(1):105-130, 2015.

[24] G. D. Abowd e E. D. Mynatt. 2000. Charting past, present, and future research in ubiquitous computing. ACM Transactions on Computer- 
Human Interaction (TOCHI). 7(1):29-58, 2000.

[25] M. d. G. Pimentel, Y. Ishiguro, B. Kerimbaev, G. D. Abowd e M. Guzdial. Supporting Educational Activities through Dynamic Web Interfaces. Interacting with Computers. 13(3):353-374, 2001.

[26] B. Efron. Bootstrap Methods: Another Look at the Jackknife. The Annals of Statistics. 7(1):1-26, 1979.

[27] I. de Barros e M. Tavares. Estimativa do Tamanho Ótimo de Parcelas Experimentais Através de Cálculos Algébricos. Bragantia. 54(1):209-215, 1995.

[28] D. J. Ozer. Correlation and the Coefficient of Determination. Psychological Bulletin. 97(2): 307-315, 1985.

[29] ABNT. NBR 5891: Regras de Arredondamento na Numeração Decimal. NBR 4: Norma Brasileira Probatória. Pág. 1, 1977.

[30] M. Last. Improving Data Mining Utility with Projective Sampling. Proc. of the $15^{\text {th }}$ ACM International Conference on Knowledge Discovery and Data Mining. Págs. 487-496, 2009.

[31] L. Pelkowitz e S. C. Schwarts. Asymptotically Optimum Sample Size for Quickest Detection. IEEE Transactions on Aerospace and Electronic Systems. 23(2):263-272, 1987.

[32] Y.-J. Cui, S. Davis, C.-K. Cheng e X. Bai. A Study of Sample Size with Neural Network. Em Proceedings of the 2004 International Conference on Machine Learning and Cybernetics. Págs. 3444-3448, 2004.

[33] J. H. Eu. A Sampling Approach to Real-Time Performance Monitoring of Digital Transmission Systems. Em $8^{\text {th }}$ Annual International Phoenix Conference on Computers and Communications. Págs. 207-211, 1989.

[34] H. Pham. Optimal Design of life Testing for ULSI Circuit Manufacturing. IEEE Transactions on Semiconductor Manufacturing. 5(1):68-70, 1992.
[35] R. Confalonieri, M. Acutis, G. Bellocchi e G. Genovese. Resampling-based Software for Estimating Optimal Sample Size. Environmental Modelling \& Software, 22(12):1796-1800, 2007.

[36] P. Müller, G. Parmigiani, C. Robert e J. Rousseau. Optimal Sample Size for Multiple Testing: the Case of Gene Expression Microarrays. Journal of the American Statistical Association, 99(468):990-1001, 2004.

[37] F. Yuan, J. Lu e B. M. Batalden. Sample Size Determination and Confidence Interval Derivation for Exponential Distribution. Em Proc. of the $26^{\text {th }}$ International Ocean and Polar Engineering Conference. Págs. 664-669, 2016.

[38] J. Nielsen. Why You Only Need to Test With 5 Users. Jakob Nielsen's Alertbox, 2000. https://www.nngroup.com/articles/why-you-onlyneed-to-test-with-5-users/. Acesso em Fevereiro/2017.

[39] W. Hwang e G. Salvendy. Number of People Required for Usability Evaluation: The $10 \pm 2$ Rule. Communications of the ACM. 53(5):130133,2010

[40] M. Schmettow. Sample Size in Usability Studies. Communications of the ACM. 55(4):64-70, 2012.

[41] D. M. Mertens. Research and Evaluation in Education and Psychology: Integrating Diversity With Quantitative, Qualitative, and Mixed Methods. SAGE Publications, 2014.

[42] A. J. Onwuegbuzie e K. M. T. Collins. A Typology of Mixed Methods Sampling Designs in Social Science Research. Qualitative Report. 12(2):281-316, 2007.

[43] R. Slavin e D. Smith. The Relationship Between Sample Sizes and Effect Sizes in Systematic Reviews in Education. Educational Evaluation and Policy Analysis. 31(4):500-506, 2009. 